NOTES AND CORRESPONDENCE

\title{
On the Meridional Structure of Annular Modes
}

\author{
Matthew A. H. Wittman And Andrew J. Charlton \\ Department of Applied Physics and Applied Mathematics, Columbia University, New York, New York \\ LORENZO M. POlvani \\ Department of Applied Physics and Applied Mathematics, and Department of Earth and Environmental Sciences, Columbia \\ University, New York, New York
}

(Manuscript received 26 April 2004, in final form 22 September 2004)

\begin{abstract}
Using a simple stochastic model, the authors illustrate that the occurrence of a meridional dipole in the first empirical orthogonal function (EOF) of a time-dependent zonal jet is a simple consequence of the north-south excursion of the jet center, and this geometrical fact can be understood without appealing to fluid dynamical principles. From this it follows that one ought not, perhaps, be surprised at the fact that such dipoles, commonly referred to as the Arctic Oscillation (AO) or the Northern Annular Mode (NAM), have robustly been identified in many observational studies and appear to be ubiquitous in atmospheric models across a wide range of complexity.
\end{abstract}

\section{Introduction}

Attempts to explain intraseasonal variability in the Northern Hemisphere have recently popularized a large-scale pattern known as the Arctic Oscillation (AO) and the related Northern Annular Mode (NAM). Diagnostics based upon the AO/NAM are now widely used in many fields of the geophysical sciences as a convenient way of describing the atmospheric variability.

With respect to its temporal evolution, it is well established that no specific periodicity is associated with this phenomenon, in that no frequency peaks appears in the power spectrum of the AO/NAM. With respect to its spatial structure, in contrast, a very robust pattern consistently emerges, independently of the specifics of the AO/NAM calculation (Baldwin and Dunkerton 1999; Baldwin and Dunkerton 2001; Lorenz and Hartmann 2003; Thompson and Wallace 1998). This pattern appears as a roughly axisymmetric meridional dipole.

Corresponding author address: M. A. H. Wittman, 500 W. 120th Street, Room 200, Columbia University, New York, NY 10027. E-mail: maw2006@columbia.edu
Why this pattern should be dipolar is the subject of this short note.

The question is made even more compelling in view of recent results from numerical models. Almost without exception, a dipolar AO/NAM pattern is found in nearly all numerical models, across a wide range of complexity, from comprehensive general circulation models (Christiansen 2000; Fyfe et al. 1999; Norton 2003; Shindell et al. 1999), to aquaplanet models (Cash et al. 2003; Feldstein and Lee 1996), to dry dynamicalcore models (Polvani and Kushner 2002), all the way to extremely idealized single-layer models (Tanaka 2003; Vallis et al. 2004), and even the random-walk, momentum-conserving jet of Gerber and Vallis (2005).

The ubiquity of this dipolar pattern, in both observational and modeling studies, suggests that its presence must to be related to some relatively gross common feature. The obvious candidate is the presence of a time dependent zonal jet. In this note we illustrate how, irrespective of the details of the underlying dynamics, the first empirical orthogonal function (EOF) will appear as a dipole, provided the bulk of the jet variability resides in meridional excursions of the jet center. 

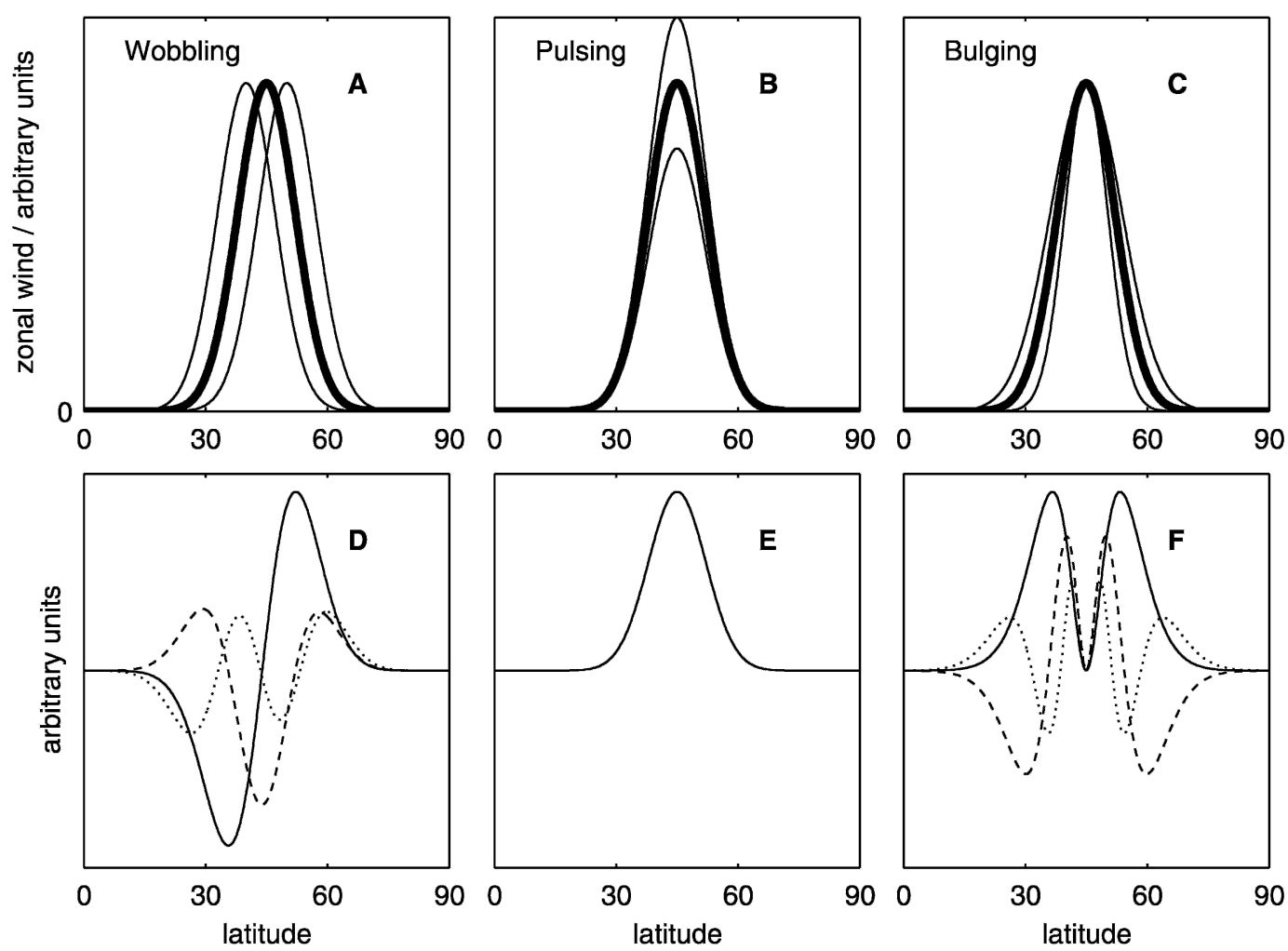

FIG. 1. (a)-(c) Schematic illustration of the three types of jet variability, as described by Eqs. (1)-(4). Thick lines represent the mean jet profile and thin lines show a jet one standard deviation away from its mean. (d)-(f) Corresponding EOFs resulting from integration of the model with only one jet parameter varying. The EOFs are ordered by the amount of total variance they explain. In each case the first EOF is the solid line, the second is dashed, and the third is dotted (pulsing produces only one EOF). The units on the ordinate and the relative amplitudes of the EOFs are arbitrary.

\section{Method}

To illustrate the relationship between different types of zonal jet variability and the resulting EOFs we use a simple stochastic model. Following Fyfe (2003), our model consists of a zonal jet, which is represented by a simple function of latitude and time $u(\phi, t)$ as follows:

$$
u(\phi, t)=U(t) \exp \left\{-\left(\frac{\phi-\Phi(t)}{H(t)}\right)^{2}\right\} .
$$

The jet variability is a result of the time dependence of the three jet parameters: the mean position $\Phi$, the strength $U$, and the width $H$. These are represented as Ornstein-Uhlenbeck stochastic processes, and their variation is described by the combination of pure noise and relaxation to a mean value. The three equations for $H, U$, and $\Phi$ are, therefore,

$$
\begin{aligned}
& d \Phi=-b_{1}\left(\Phi-\Phi_{0}\right) d t+\sigma_{1} d W_{t}, \\
& d U=-b_{2}\left(U-U_{0}\right) d t+\sigma_{2} d W_{t}, \\
& d H=-b_{3}\left(H-H_{0}\right) d t+\sigma_{3} d W_{t},
\end{aligned}
$$

where $W_{t}$ is a Wiener process (e.g., Karatsas and Shreve 1997) with a different realization for each jet parameter; $U_{0}, \Phi_{0}$, and $H_{0}$ are the mean values of the jet parameters; the coefficients $b_{1}, b_{2}$, and $b_{3}$ represent the strength of the relaxation to the mean values; and the coefficients $\sigma_{1}, \sigma_{2}$, and $\sigma_{3}$ the strength of the noise forcing the three jet parameters. Following Vallis et al. (2004), we label variations in the latitudinal position of the jet as wobbling, variations in the strength of the jet as pulsing, and variations in the width of the jet as bulging. These three types of variability are illustrated schematically in the top row of Figs. 1a-c.

We numerically integrate Eqs. (2)-(4) with an explicit Euler scheme, which, due to the simplicity of the stochastic processes, is equivalent to the higher order Milstein scheme and thus converges strongly with order $\Delta t$. The time step is 0.05 days, and the integration length is 1000 days. At each time, the values of the jet parameters are used to compute the zonal wind at all latitudes from the specified jet function (1). From the resultant time series of wind at each latitude, we compute the first three EOFs of the jet variability. 


\section{Results}

Consider first the EOFs produced by the individual variation of the jet parameters, as illustrated in the bottom row of Fig. 1. The EOFs in each panel are computed from a time series obtained by setting $b_{i}=\sigma_{i}=$ 1 for each of Eqs. (2)-(4) in turn, and setting $b_{i}=\sigma_{i}=$ 0 for the other two. The point of this exercise is to examine the shape of the EOFs due to each possible jet variation (wobbling, pulsing, or bulging), and in particular the symmetry of these EOFs about the jet center.

Figure 1d shows EOFs produced by wobbling alone. The first EOF (solid line) is dipolar and AO/NAM-like, with the crossing point located at the jet center. Note that the EOFs produced by wobbling are alternately symmetric and antisymmetric about the jet center. Figure 1e shows the EOFs produced by pulsing alone. In this case, the first and only EOF is monopolar, with an identical shape to the jet itself, and is thus symmetric about the jet center. Finally, Fig. 1f shows the EOFs produced by bulging. Note that all EOFs in this case are more complex than a simple dipole, and more importantly, they are all symmetric about the jet center.

From the above, we conclude that only variation in the mean jet position (i.e., wobbling) is capable of producing EOFs that are dipolar and antisymmetric about the mean jet position. This suggests that the dipolar meridional structure of the AO/NAM is likely due to variation in the mean position of the jet. We now show that when our stochastic model is forced with realistic values for the coefficients, designed to simulate Northern Hemisphere zonal mean wind variability, the first EOF is indeed dipolar and is due to the dominance of variability in the position of the jet over variability in its strength and width.

To accomplish this, Eqs. (2)-(4) are integrated with the values given in Table 1 . These values are obtained from the National Centers for Environmental Prediction-National Center for Atmospheric Research (NCEP-NCAR) 1000-hPa November-March daily zonal mean zonal wind data from 1958 to 2001 (Kalnay et al. 1996), by fitting the Gaussian jet function (1) to the zonal wind profile on each day, and obtaining the variance and autocorrelation $e$-folding time of each jet

TABLE 1. Realistic model coefficients, obtained from the NCEP-NCAR reanalysis, as explained in the text.

\begin{tabular}{llcl}
\hline \hline & Mean values & $b\left(\right.$ day $\left.^{-1}\right)$ & $\sigma$ \\
\hline Position & $\Phi_{0}=45^{\circ} \mathrm{N}$ & $1 / 3$ & 4.8 \\
Amplitude & $U_{0}=5 \mathrm{~m} \mathrm{~s}^{-1}$ & $1 / 4$ & 0.92 \\
Width & $H_{0}=10^{\circ}$ & $1 / 2$ & 2.7 \\
\hline
\end{tabular}

parameter from the resulting time series. These observationally derived values are related to the coefficients $b_{i}$ and $\sigma_{i}$ by the relationships

$$
\operatorname{autocorrelation}(i)=1 / b_{i} \text { and } \operatorname{variance}(i)=\sigma_{i}^{2} /\left(2 b_{i}\right),
$$

where $i$ represents each of the jet parameters $(\Phi, U, H)$ in turn. For each jet parameter, therefore, the value of $b_{i}$ is obtained from the autocorrelation as computed from the data and, from the variance, the value of $\sigma_{i}$ immediately follows.

The EOFs resulting from integration of (2)-(4) with the realistic coefficient values of Table 1 are shown in Fig. 2. Not surprisingly, the first EOF is a dipole, qualitatively identical to the dipole obtained by a mere wobbling of the jet (Fig. 1d), and representing $50 \%$ of the total variance. As is illustrated above, only variations in the north-south position of the jet can produce such an antisymmetric dipole.

\section{Discussion}

Using a simple model of zonal jet variability we have shown that the familiar dipolar structure of the first EOF is a direct and very simple consequence of the variability in the north-south position of the jet, as the EOFs corresponding to other forms of variability do not posses the required symmetry across the jet maximum. From this, therefore, we argue that the ubiquity

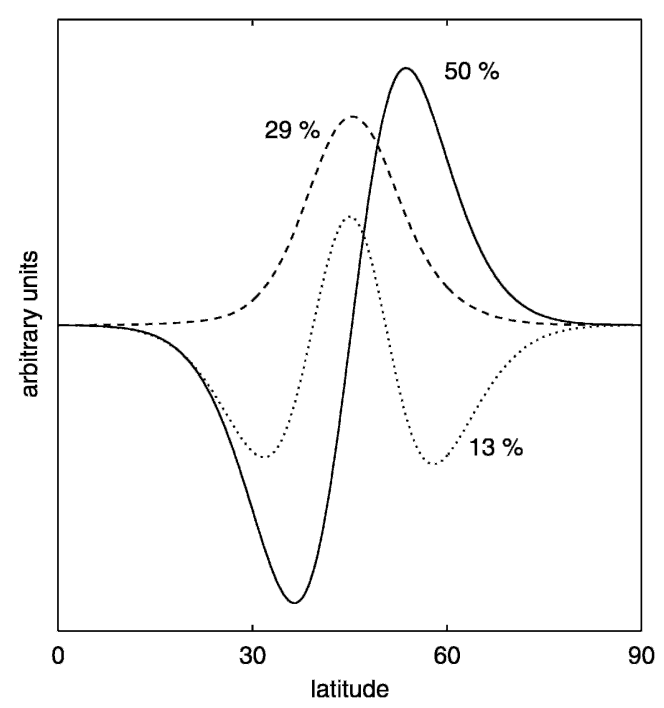

FIG. 2. The first three EOFs resulting from the integration of (2)-(4) using the realistic coefficient values in Table 1, obtained from the NCEP-NCAR reanalysis. The first EOF is the solid line, the second the dashed line, and the third the dotted line, as in Fig. 1. The percentage of the explained variance is indicated next to each curve. 
of a dipolar first EOF across a wide range of models is perhaps not terribly surprising. Even a low-resolution model will be able to reproduce an AO/NAM-like dipole as long as it is able to resolve (however crudely) a midlatitude jet and its north-south variability.

It should also be noted that the study of the northsouth variability of atmospheric jets has a long history, in the context of the so-called zonal index. In particular, as shown by Deweaver and Nigam (1999), the correlation between zonal index time series and the first EOF of zonal wind variability is very high $(-0.79)$. The illustration given above from our simple model serves the purpose of clarifying the fact that the dipolar structure of the first EOF is due to the wobbling of the jet about its mean position, and not to other types of variability.

Finally, the results from our simple model raise an interesting question about the nature of variability of the extratropical jet: why is this variability dominated by changes to the meridional position of the jet, as opposed to jet strengthening/weakening or bulging/ contracting? At first glance, one might suspect that the answer to this would be related to the nature of baroclinic eddies and their interaction with the mean flow. However, similar dipolar structures in the first EOF appear in purely barotropic models (Vallis et al. 2004) and in studies of variability in the stratosphere (Baldwin and Dunkerton 1999), in which cases jet variability is surely dominated by different processes. This question clearly deserves further study.

Acknowledgments. We are grateful to R. Saravanan, Jim Hansen, Geoff Vallis, Ed Gerber, and John Fyfe for useful discussions, and for constructive criticism on an earlier version of this note. This work is funded, in part, by a grant from the National Science Foundation.

\section{REFERENCES}

Baldwin, M. P., and T. J. Dunkerton, 1999: Propogation of the Arctic Oscillation from the stratosphere to the troposphere. J. Geophys. Res., 104, 30 937-30946.
— , and — 2001: Stratospheric harbingers of anomalous weather regimes. Science, 294, 581-584.

Cash, B. A., P. J. Kushner, and G. K. Vallis, 2003: The structure and composition of annular modes in an aquaplanet general circulation model. J. Atmos. Sci., 59, 3399-3414.

Christiansen, B., 2000: A model study of the dynamical connection between the Arctic Oscillation and stratospheric vacillations. J. Geophys. Res., 105, 29 461-29 474.

Deweaver, E., and S. Nigam, 1999: Do stationary waves drive the zonal mean jet anomalies of the northern winter. J. Climate, 13, 2160-2176.

Feldstein, S., and S. Lee, 1996: Mechanisms of zonal index variability in an aquaplanet GCM. J. Atmos. Sci., 53, 3541-3555.

Fyfe, J. C., 2003: Separating extratropical zonal wind variability and mean change. J. Climate, 16, 863-874.

- G. J. Boer, and G. M. Flato, 1999: The Arctic and Antarctic Oscillations and their projected changes under global warming. Geophys. Res. Lett., 26, 1601-1604.

Gerber, E. P., and G. K. Vallis, 2005: A stochastic model for the spatial structure of annular patterns of variability and the North Atlantic Oscillation. J. Climate, 18, 2102-2118.

Kalnay, E., and Coauthors, 1996: The NCEP/NCAR 40-Year Reanalysis Project. Bull. Amer. Meteor. Soc., 77, 437-471.

Karatsas, I., and S. Shreve, 1997: Brownian Motion and Stochastic Calculus. $2 \mathrm{~d}$ ed. Springer-Verlag, $470 \mathrm{pp}$.

Lorenz, D. J., and D. L. Hartmann, 2003: Eddy-zonal flow feedback in the Northern Hemisphere winter. J. Climate, 16, 1212-1227.

Norton, W. A., 2003: Sensitivity of Northern Hemisphere surface climate to simulation of the stratospheric polar vortex. Geophys. Res. Lett., 30, 1627, doi:10.1029/2003GL016958.

Polvani, L. M., and P. J. Kushner, 2002: Tropospheric response to stratospheric perturbations in a relatively simple general circulation model. Geophys. Res. Lett., 29, 14 284-14 287.

Shindell, D. T., R. L. Miller, G. A. Schmidt, and L. Pandolfo, 1999: Simulation of recent northern winter climate trends by greenhouse-gas forcing. Nature, 399, 452-455.

Tanaka, H. L., 2003: Analysis and modeling of the Arctic Oscillation using a simple barotropic model with baroclinic eddy forcing. J. Atmos. Sci., 60, 1359-1379.

Thompson, D. W. J., and J. M. Wallace, 1998: The Arctic Oscillation signature in the wintertime geopotential height and temperature fields. Geophys. Res. Lett., 25, 1297-1300.

Vallis, G. K., E. P. Gerber, P. J. Kushner, and B. J. Cash, 2004: A mechanism and simple dynamical model of the North Atlantic Oscillation and annular modes. J. Atmos. Sci., 61, 264280. 